\title{
ANALYSIS OF COMPUTATIONAL ERROR BEHAVIOR AT THE NUMERICAL SIMULATION OF CURVED ELECTRODYNAMIC SYSTEMS
}

\author{
Artem Badarin \\ Neuroscience and Cognivite Technology Laboratory \\ Center for Technologies in Robotics and Mechatronics Components \\ Innopolis University \\ Russia \\ Faculty of Nonlinear Processes \\ Saratov State University \\ Russia \\ A.Badarin@innopolis.ru
}

Article history:

Received 02.10.2019, Accepted 05.12.2019

\begin{abstract}
The paper presents an analysis of the approach to the numerical study of vacuum microwave electronic systems by finite-difference time-domain (FDTD) method. The results on approximating the boundary between two materials are presented. The influence of changes in the geometric shape of the resonator on the frequency obtained in the numerical experiment is analyzed. The sensitivity of the boundary approximation methods to small changes in geometric parameters is investigated.
\end{abstract}

\section{Key words}

PIC; FDTD; numerical simulation; optimization; calculation error; boundary approximation

\section{Introduction}

One of the most effective methods for studying vacuum and plasma electronics systems is numerical simulation using modern computing technologies[Birdsall and Langdon, 2005; Kurkin et al., 2018; Aminov and Ovsyannikov, 2015]. The most perfect and demanded at the moment here are three-dimensional fully electromagnetic codes, in which Maxwell equations are numerically solved to find self-consistent electromagnetic fields in the system, and the large particle method (PIC-method) is used to model the dynamics of charged particle beam [Birdsall and Langdon, 2005; Kurkin et al., 2018; Altsybeyev et al., 2016; Rashchikov, 2014; Polozov and Rashchikov, 2018; Camporeale et al., 2016; Bottino and Sonnendrücker, 2015; Frolov et al., 2016; Badarin et al.,
2018]. This approach complements well and replaces to some extent experimental research, especially at the stages of development of prototypes of new devices, as well as optimization of existing ones. The calculation of the intrinsic electromagnetic fields of the electron beam and electrodynamic systems is based on the joint solution of the Vlasov kinetic equation for the electron distribution function and Maxwell equations.

The essence of the method is to divide the problem into two parts: the solution of the kinetic equation is modeled using a large number $\mathrm{N}$ of "large" particles with the same charge-to-mass ratio; the solution to the field part of the problem is often carried out using the Yee algorithm [Yee, 1966; Taflove and Hagness, 2005]. In turn, this paper devoted to a detailed examination of the field part of this problem and to the analysis of the arising computational errors.

\section{Boundary Approximation Methods}

One of the most important issues when using the finite difference time domain method (FDTD) is the approximation of curved boundaries on a computational grid. Here, the simplest way is the so-called staircase approximation of the boundary (fig. 1a). In particular, the fig. 1a represents an approximation of the boundary between a perfect electric conductor (PEC) and a vacuum. In this representation, the curved border is replaced by its staircase counterpart, so that each grid cell is uniformly filled with only one type of material. In this case, the nodes of the computational grid located inside the PEC, as well as the nodes corresponding to the tangential components 

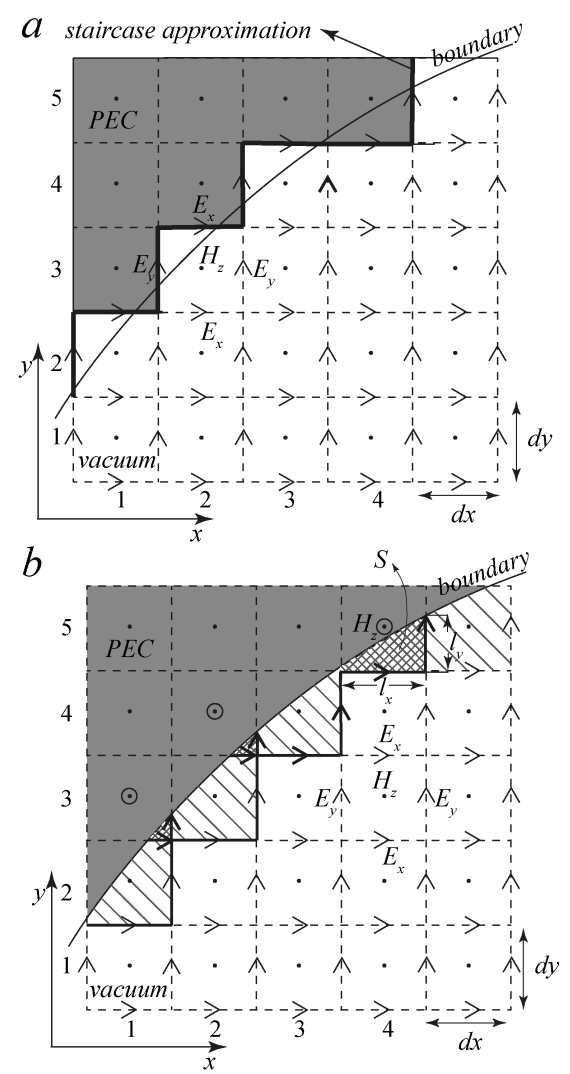

Figure 1. Representation of the computational grid for the (a) staircase and $(b)$ Dey-Mittra approximation.

of the electric field strength in the approximated geometry, are not calculated, since they are equal to zero due to boundary conditions and the absence of the field inside the PEC.

Such approximation of the boundary is well suited for simulation systems with rectangular geometry but, at the same time, despite its simplicity, it allows simulation of the systems with curvilinear geometry with good accuracy.

Currently, several approaches to the approximation of curvilinear geometry have been developed, which can be classified into two groups. These are methods requiring the nonorthogonal coordinates or totally unstructured grids, and methods that deform the Cartesian computational grid only locally to take into account surface curvature [Palandech et al., 1992; Gedney and Roden, 2000; Taflove and Hagness, 2005; Railton and Schneider, 1999].

It should be noted that the use of a global nonorthogonal grid leads to great difficulties when using the PIC method. Because of this feature, methods involving only a locally deformed mesh are more preferable in the context of the problem under consideration and often have a simpler implementation and high accuracy.

The group of methods that locally deform the mesh includes method called "Contour path finite-difference time-domain" (CPFDTD) [Jurgens and Taflove, 1993]. The essence of this method is to change the contour of the cell that the PEC crosses so that the distorted contour exactly corresponds to the free space of this cell (cells $(1,2),(2,3),(3,4)$ in fig. 1b). This approach allows taking into account the curvature of the surface crossing the cell during integration. This method approximates geometry with high accuracy but can be unstable due to the need to borrow the nearest components of the electric field. It should be noted that there is the modernization of this method making it stable but increasing computational complexity [Railton and Craddock, 1996].

At the same time, S. Dey and R. Mittra proposed the method characterized by high accuracy, and stability, and an insignificant increase in computational complexity relative to the staircase approximation of the boundary [Dey and Mittra, 1997]. The essence of this method is to include in the calculation of cells, the free space of which is more than $1.5 \%$ of the area of the undistorted cell, and the ratio between the largest side of the distorted cell (for the cell $(4,5)$ in fig. $1 \mathrm{~b}$ it is $\max \left(l_{x}, l_{y}\right)$ ) and its area was less than 10 . This leads to the calculation of the grid nodes for the magnetic field that is inside a conductor (for example, cells $(1,3),(2,4),(4,5)$ in fig. 1b). It should be noted that this approach requires a decrease in the time step in order to maintain stability. In particular, for the parameters $1.5 \%$ and 10 , the time step should be two times less than the step determined by the Courant number [Dey and Mittra, 1997].

In this paper the results on approximating the boundary between two materials are presented. The influence of changes in the geometric shape of the resonator on the frequency and error obtained in the numerical experiment is analyzed.

\section{Results}

As a test problem, the frequency of a cylindrical resonator was investigated as a function of its radius. At the initial time moment, the distribution of the electric mode $E_{111}$ was set in space; then frequency of electromagnetic field oscillations was measured by the Fourier spectrum. In this paper, the space step was uniform and equal to $\Delta=1, \mathrm{~mm}$, the time step was chosen equal to $\Delta t=k_{\text {stable }} * \Delta t_{\text {Courant }}$, where $k_{\text {stable }}=0.25$. The cavity radius was changed with the step of $0.1 \mathrm{~mm}$ in the range from 7 to $22 \mathrm{~mm}$ fig. $2 \mathrm{~b}$ shows the dependence of the resonance frequency on the radius of the system under study. Here and below, the red line corresponds to the staircase approximation, the green line is the approximation using the method proposed by S. Dey and R. Mittra, and the black is the theoretically calculated frequency. It is clearly seen that each of the methods determines the resonant frequency well.

Consider an enlarged fragment of this dependence, which is shown in fig. 2a. It can be seen that for the staircase approximation, the frequency change with increasing radius occurs sharply and around the theoretically calculated value. The frequency determined using the Dey-Mittra method is characterized by a smooth change 

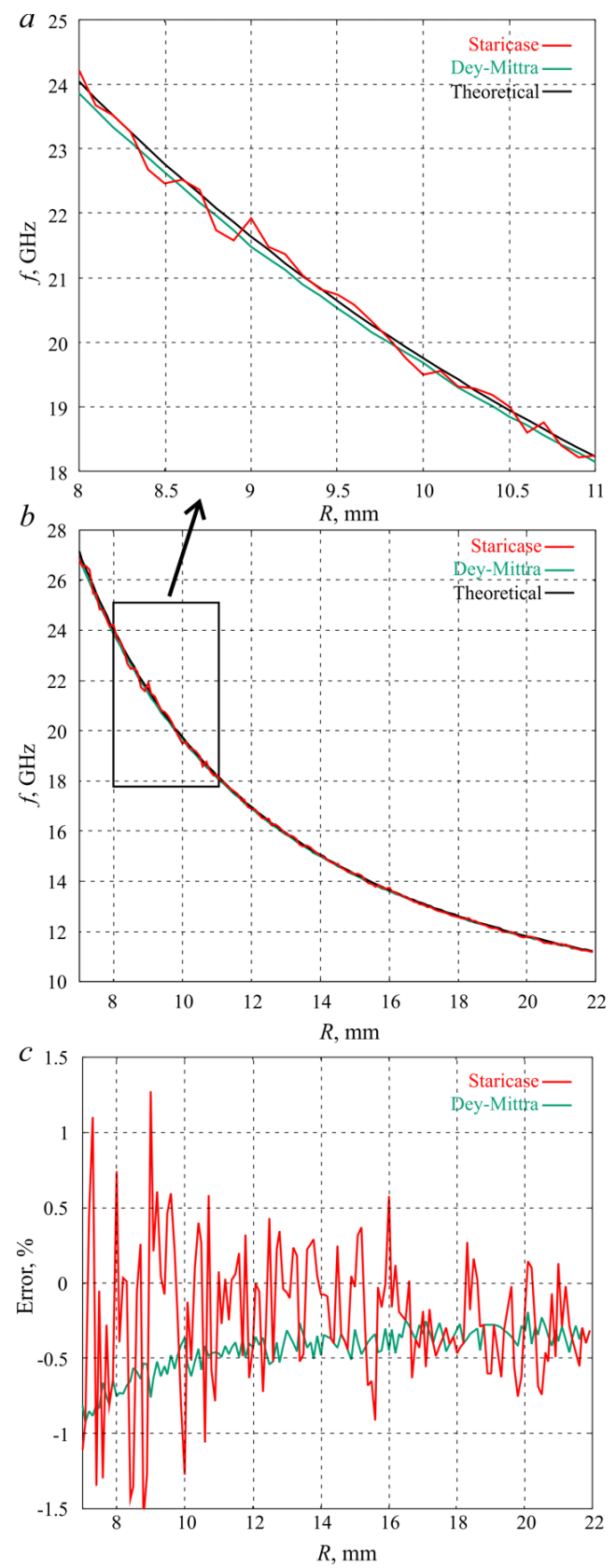

Figure 2. Comparison of the dependence of frequency on the radius for the staircase and Dey-Mittra approximation $(b)$; enlarged fragment (a); computational error $(c)$.

with increasing radius. At the same time, the curve obtained using this method is always below the theoretical curve. This fact is understandable from a physical point of view. Indeed, since this method involves the use of grid points lying inside the conductor, that leads to a phantom increase in the geometric size of the cavity on the computational grid and, as a result, to a decrease in the resonance frequency.

Fig. 2c illustrates the dependence of the frequency measurement error on the cavity radius for the two considered methods. It can be seen that for both methods, the computational error decreases with an increase in the cavity radius. Such dynamic is associated both with the

increase in the number of cells per wavelength and with the decrease in the curvature of the approximated surface.

Note, that for the analysis of a microwave device, it is often important to investigate the effect of small changes in geometric parameters on the electrodynamic characteristics of the system. In this regard, we turn to a detailed analysis of the dynamics of the error in determining the frequency in order to study the possibility of reliable detection of small changes using the methods considered.

To do this, it is required to approximate the curves $(f(R))$, obtained in the numerical experiment by a power polynomial (in this paper, the polynomial of degree 10 $f_{\text {app }}(R)$ ) was built by the least-squares method). Then it is necessary to subtract their power approximation from the experimental curves:

$$
\xi(R)=f(R)-f_{\text {app }}(R)
$$

The $\xi(R)$ curves obtained in this way reflect deviations of the measured frequency on the radius for each of the methods (see fig. 3a). It is clearly seen that the deviations obtained using the Dey-Mittra method are much smaller than the deviations for the staircase approximation. At the same time, for the staircase approximation, the fast decrease in $\xi(R)$ is observed with increasing radius and, accordingly, the number of cells per wavelength.

To analyze the dynamics of $\xi(R)$, a standard deviation $\sigma(R)$ was constructed in the window of width $\delta R_{w}=4 \Delta$ for the distribution of $\xi(R)$ (see fig. $3 b$ ). Here, the standard deviation is given on a logarithmic scale in view of the different scales of the studied quantities. It is clearly seen that $\sigma_{s}(R)$ and $\sigma_{d}(R)$ decrease monotonically with increasing $R$. At the same time, $\sigma_{s}(R)$ exceeds $\sigma_{d}(R)$ by more than an order of magnitude for small $\mathrm{R}$ and approaches $\sigma_{d}(R)$ with increasing $\mathrm{R}$.

\section{Conclusion}

In this paper, we considered the dynamics of the computational error in determining the frequency of a cylindrical resonator. The detailed comparison of the error dynamics for two methods of approximating the boundaries (staircase and Dey-Mittra approximation) for the FDTD method was carried out. It is shown that the error in determining the resonator frequency does not exceed $1.5 \%$ for the staircase approximation, and $0.8 \%$ for the Dey-Mittra method. Moreover, for both methods, the error decreases with an increasing radius. It is shown that using the Dey-Mittra method, it is possible to detect frequency changes by an order of magnitude more accurate than when using the staircase approximation due to smaller noise fluctuations of the frequency determination (fig. 3). At the same time, it should be noted that the maximum errors for the methods under consideration differ only by half. 

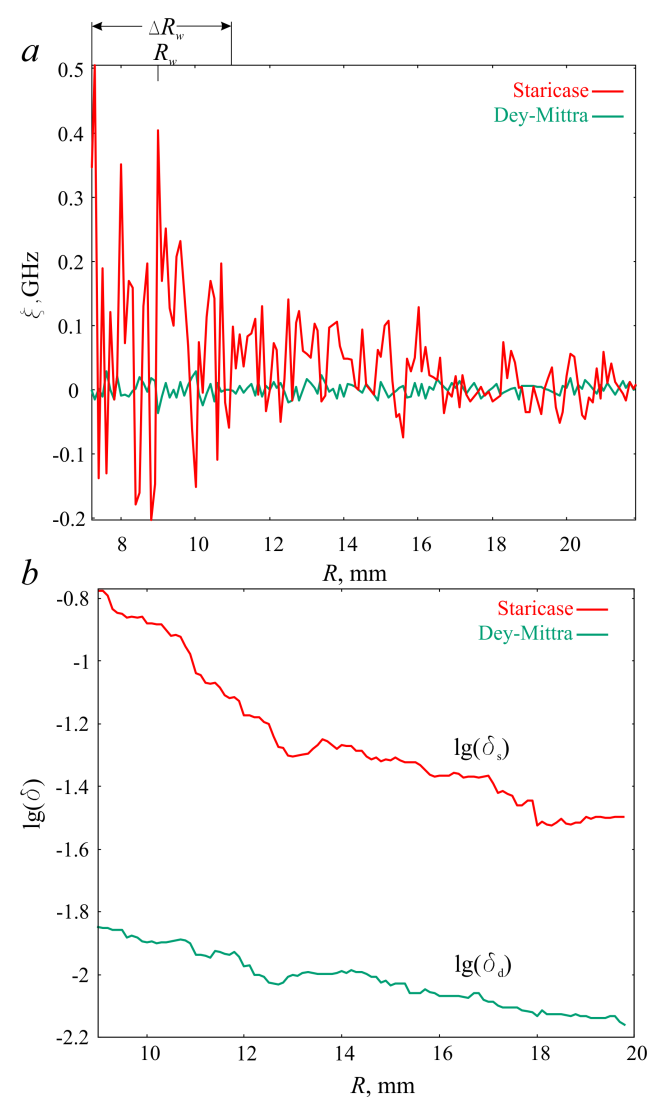

Figure 3. Distributions of the deviations of the measured frequency on the radius for the staircase and Dey-Mittra approximation $(a)$ and the dependencies of the standard deviation on the radius for these distributions $(b)$.

\section{Acknowledgements}

Artem Badarin thanks Semen Kurkin and Alexander Hramov for productive discussions. The studies were supported by the Ministry of Education and Science of the Russian Federation (Project 3.859.2017/4.6) and the Russian Foundation for Basic Research (Grant 18-3220135).

\section{References}

Altsybeyev, V. et al. (2016). On improving the particlein-cell simulations accuracy for sources of charged particles. Cybernetics and physics, 5 (1), pp. 16-20.

Aminov, R. and Ovsyannikov, A. (2015). Modeling of plasma breakdown conditions in iter. In 7th International Conference on Physics and Control (PhysCon 2015) IPACS Electronic library http://lib. physcon. ru.

Badarin, A. A., Kurkin, S. A., Koronovskii, A. A., Hramov, A. E., and Rak, A. O. (2018). Processes of virtual cathodes interaction in multibeam system. Physics of Plasmas, 25 (8), pp. 083110.

Birdsall, C. K. and Langdon, A. B. (2005). Plasma physics via computer simulation. Taylor and Francis Group.

Bottino, A. and Sonnendrücker, E. (2015). Monte carlo particle-in-cell methods for the simulation of the vlasov-maxwell gyrokinetic equations. Journal of Plasma Physics, 81 (5).

Camporeale, E., Delzanno, G. L., Bergen, B., and Moulton, J. D. (2016). On the velocity space discretization for the vlasov-poisson system: Comparison between implicit hermite spectral and particle-in-cell methods. Computer Physics Communications, 198, pp. 47-58.

Dey, S. and Mittra, R. (1997). A locally conformal finitedifference time-domain (fdtd) algorithm for modeling three-dimensional perfectly conducting objects. IEEE Microwave and Guided Wave Letters, 7 (9), pp. 273275.

Frolov, N. S., Kurkin, S. A., Khramova, M. V., Badarin, A. A., Koronovskii, A. A., Pavlov, A. N., and Hramov, A. E. (2016). Perspective sub-thz powerful microwave generator "nanovircator" for t-rays biomedical diagnostics. Proceedings of SPIE, 9917, pp. 991721-1991721-8.

Gedney, S. D. and Roden, J. A. (2000). Numerical stability of nonorthogonal fdtd methods. IEEE Transactions on Antennas and Propagation, 48 (2), pp. 231-239.

Jurgens, T. G. and Taflove, A. (1993). Threedimensional contour fdtd modeling of scattering from single and multiple bodies. IEEE Transactions on Antennas and Propagation, 41 (12), pp. 1703-1708.

Kurkin, S., Badarin, A., Koronovskii, A., Frolov, N., and Hramov, A. (2018). Modeling instabilities in relativistic electronic beams in the cst particle studio environment. Mathematical Models and Computer Simulations, 10(1), pp. 59-68.

Palandech, R., Mittra, R., et al. (1992). Modeling three-dimensional discontinuities in waveguides using nonorthogonal fdtd algorithm. IEEE Transactions on Microwave Theory and Techniques, 40 (2), pp. 346352.

Polozov, S. M. and Rashchikov, V. I. (2018). Longitudinal motion stability of electrons inside the plasma channel of lpwa. Cybernetics and Physics, 7(4), pp. 228-232.

Railton, C. and Craddock, I. (1996). Stabilised cpfdtd algorithm for the analysis of arbitrary $3 \mathrm{~d}$ pec structures. IEE Proceedings-Microwaves, Antennas and Propagation, 143 (5), pp. 367-372.

Railton, C. J. and Schneider, J. B. (1999). An analytical and numerical analysis of several locally conformal fdtd schemes. IEEE Transactions on Microwave Theory and Techniques, 47 (1), pp. 56-66.

Rashchikov, V. I. (2014). Computer simulation of residual gas ionization in gas-filled neutron tubes. Cybernetics and Physics, 3 (2), pp. 73-78.

Taflove, A. and Hagness, S. C. (2005). Сотриtational electrodynamics: the finite-difference timedomain method. Artech house.

Yee, K. (1966). Numerical solution of initial boundary value problems involving maxwell's equations in isotropic media. IEEE Transactions on antennas and propagation, 14 (3), pp. 302-307. 